\section{(1) \\ CrossMark}

\title{
Interdisciplinary multimodality management of stage III nonsmall cell lung cancer
}

\author{
Rudolf M. Huber (10) ${ }^{1}$, Dirk De Ruysscher ${ }^{2}$, Hans Hoffmann ${ }^{3}$, Simone Reu ${ }^{4}$ and \\ Amanda Tufman ${ }^{1}$
}

Affiliations: ${ }^{1}$ Division of Respiratory Medicine and Thoracic Oncology, Dept of Medicine, University of Munich Campus Innenstadt, and Thoracic Oncology Centre Munich, Member of the German Centre of Lung Research, Munich, Germany. ${ }^{2}$ Maastricht University Medical Center, Dept of Radiation Oncology (MAASTRO clinic), GROW School for Oncology and Developmental Oncology, Maastricht, The Netherlands. ${ }^{3}$ Division of Thoracic Surgery, Technical University of Munich, Munich, Germany. ${ }^{4}$ Institute of Pathology, University of Würzburg, Würzburg, Germany.

Correspondence: Rudolf M. Huber, Sektion Pneumologie und Thorakale Onkologie, Klinikum der LMU München, Lungentumorzentrum München, Ziemssenstraße 1, 80336 Munich, Germany. E-mail: huberlamed. uni-muenchen.de

@ERSpublications

Stage III NSCLC is a heterogenous group. Outcome depends on a good inter- and multidisciplinary strategy. Local treatment and systemic therapy both have to be optimised. Interdisciplinary trials integrate systemic targeted therapy or immunotherapy. bit.ly/2WstXtX

Cite this article as: Huber RM, De Ruysscher D, Hoffmann $\mathrm{H}$, et al. Interdisciplinary multimodality management of stage III nonsmall cell lung cancer. Eur Respir Rev 2019; 28: 190024 [https://oi.org/ 10.1183/16000617.0024-2019].

ABSTRACT Stage III nonsmall cell lung cancer (NSCLC) comprises about one-third of NSCLC patients and is very heterogeneous with varying and mostly poor prognosis. It is also called "locoregionally or locally advanced disease". Due to its heterogeneity a general schematic management approach is not appropriate. Usually a combination of local therapy (surgery or radiotherapy, depending on functional, technical and oncological operability) with systemic platinum-based doublet chemotherapy and, recently, followed by immune therapy is used. A more aggressive approach of triple agent chemotherapy or two local therapies (surgery and radiotherapy, except for specific indications) has no benefit for overall survival. Until now tumour stage and the general condition of the patient are the most relevant prognostic factors. Characterising the tumour molecularly and immunologically may lead to a more personalised and effective approach. At the moment, after an exact staging and functional evaluation, an interdisciplinary discussion amongst the tumour board is warranted and offers the best management strategy.

\section{Introduction}

Locally advanced nonsmall cell lung cancer (NSCLC) is classified according to the TNM staging system as stage III with subclassification into stage IIIA, IIIB and IIIC (TNM 8) [1, 2]. Prognosis for stage III disease has an intermediate position between stage I-II disease and stage IV disease. Overall, the prognosis is poor with failures occurring in the majority of the patients both locally and at distant sites. Therefore, optimal local control as well as systemic treatment are essential. Stage III patients are still a very heterogeneous group ranging from individuals presenting with multiple nodules in the lungs, tumours invading mediastinal structures, unilateral mediastinal lymph nodes and contralateral nodes without a detectable primary tumour. In view of this heterogeneity, this stage clearly needs even more than other stages an interdisciplinary

Provenance: Submitted article, peer reviewed.

Received: March 112019 | Accepted after revision: May 242019

Copyright (CERS 2019. This article is open access and distributed under the terms of the Creative Commons Attribution Non-Commercial Licence 4.0. 
multimodality approach. This approach is also recommended in international guidelines [3, 4]. One important discussion point within the multidisciplinary tumour board is the question of whether resectable stage III disease in a specific patient has better overall local tumour control with surgery and/or radiotherapy and in which sequence and combination the different modalities should be applied. Also, the possible resulting complications and patient preferences have to be considered. The reason is that when lumping all stage III patients together, the local control and overall survival is similar with only surgery or radiotherapy as the sole local therapy, but that the combination of both results in lower local failure rates without a demonstrated improvement in survival [5]. However, for individual patients, the situation may be different, although this is by no means built on hard data, with the possible exception of superior sulcus tumours. The standard of care for unresectable stage III disease is treatment with radiation therapy and platinum-based chemotherapy. Considerable effort has been directed at attempting to optimise the use of radiotherapy and chemotherapy, but the prognosis remains poor and improvements in survival over the past decade have been limited. The same lack of improvement in survival also applies for resectable disease; the definition of which is also dependent on local expertise, and which can be treated either with surgery or with radiotherapy. In the absence of differences in survival, the most important question in resectable tumours is which local therapy will result in the least short- and long-term toxicity.

With the introduction of targeted therapy and immune-checkpoint inhibitors for the systemic treatment in stage IV and the demonstrated synergism between radiotherapy and immune therapy [6], several studies have combined these agents in the multimodality treatment in stage III, resulting in the new standard of combining immune therapy with concurrent chemotherapy and radiotherapy $[7,8]$.

\section{Heterogeneity of stage III}

Stage III NSCLC is a heterogeneous group of tumours and patients with varying prognosis, tumour and nodal status, and treatment options. These tumours differ widely in their size (T1-4), as well as the extent of local extension and pattern of nodal involvement (N0-N3) (table 1). In the subcategories of lymph node classification there is still a wide variance. For example, the ipsilateral mediastinal lymph node involvement N2 ranges from a single involved lymph node at one level to multiple grossly enlarged lymph nodes already identified by computed tomography. Accordingly the prognosis for patients with stage III disease varies widely, with overall 5-year survival rates for clinical/pathological stage IIIA (TNM 7) of 19\%/24\% and for stage IIIB (TNM 7) of 7\%/9\% [9]; although there is considerable variation even within these sub-classes. In the new classification (TNM 8) prognosis varies between a 5 -year survival rate of $36 \% / 41 \%$ in clinical/ pathological stage IIIA, 26\%/24\% in stage IIIB and 13\%/12\% in stage IIIC (International Association for the Study of Lung Cancer (IASLC) global database of patients receiving a diagnosis between 1999 and 2010) (table 2) [2]. Resected N2 disease also has a very different prognosis depending on the extent of lymph node involvement [10]. 5-year survival ranges from about 35\%, if the lymph node involvement at one level

\begin{tabular}{|c|c|c|c|c|c|}
\hline $\mathrm{T} / \mathrm{M}$ and label & Description & No & N1 & N2 & N3 \\
\hline \multicolumn{6}{|l|}{ T1 } \\
\hline T1a & $\leqslant 1 \mathrm{~cm}$ & $\mathrm{~A} 1$ & II B & III A & III B \\
\hline $\mathrm{T} 1 \mathrm{~b}$ & $>1-2 \mathrm{~cm}$ & I A2 & II B & III A & III B \\
\hline T1c & $>2-3 \mathrm{~cm}$ & I A3 & II B & III A & III B \\
\hline \multicolumn{6}{|l|}{$\mathrm{T} 2$} \\
\hline \multirow[t]{2}{*}{ T2a } & Central, visceral and pleura & I B & II B & III A & III B \\
\hline & $>3-4 \mathrm{~m}$ & I B & $\| \mathrm{B}$ & III A & III B \\
\hline $\mathrm{T} 2 \mathrm{~b}$ & $>4-5 \mathrm{~cm}$ & $\| \mathrm{A}$ & II B & III A & III B \\
\hline \multirow[t]{3}{*}{ T3 } & $>5-7 \mathrm{~cm}$ & II B & III A & III B & III C \\
\hline & Invasive & II B & III A & III B & III C \\
\hline & Satellite & II B & III A & III B & III C \\
\hline \multirow[t]{3}{*}{ T4 } & $>7 \mathrm{~cm}$ & III A & III A & III B & III C \\
\hline & Invasive & III A & III A & III B & III C \\
\hline & Ipsilateral nodes & III A & III A & III B & III C \\
\hline \multicolumn{6}{|c|}{ 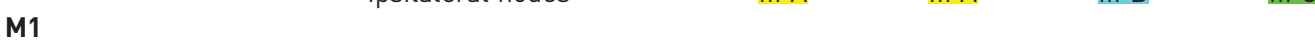 } \\
\hline \multirow[t]{2}{*}{ M1a } & Contralateral nodes & IV A & IV A & IV A & IV A \\
\hline & Pleura disseminated & IV A & IV A & IV A & IV A \\
\hline $\mathrm{M} 1 \mathrm{~b}$ & Single & IV A & IV A & IV A & IV A \\
\hline M1c & Multi & IV B & IV B & IV B & IV B \\
\hline
\end{tabular}

Modified from [1]. 
TABLE 2 5-year survival rates of the different stages: average overall survival in the International Association for the Study of Lung Cancer (IASLC) global database of patients receiving a diagnosis between 1999 and 2010

Clinical stage

Pathological stage

\begin{tabular}{lll}
\hline I A1 & 92 & 90 \\
I A2 & 83 & 85 \\
I A3 & 77 & 80 \\
I B & 68 & 73 \\
II A & 60 & 65 \\
II B & 53 & 56 \\
III A & 36 & 41 \\
III B & 26 & 24 \\
III C & 13 & 12 \\
IV A & 10 & \\
IV B & 0 & \\
\hline
\end{tabular}

Data from [2], modified from [1].

is only detected by pathology after resection, to about $5 \%$ if the ipsilateral mediastinal lymph node involvement at several levels has already been suspected with computed tomography (bulky disease).

In addition, in patients treated with chemoradiotherapy, the volume of the tumour and the extent of lymph node involvement determine the overall survival [11].

The heterogeneity of stage III is a major issue and we can only make progress if we evaluate the different subtypes separately.

\section{Relevance of histological subtypes and molecular features}

In stage IV NSCLC, the chemotherapy regimen is partly based on the histologic tumour type [12]. However, in stage III NSCLC, the PROCLAIM phase III trial failed to demonstrate a different survival in patients with stage III adenocarcinoma when concurrently treated with radiotherapy and cisplatinetoposide or cisplatin-pemetrexed [13]. This contrasts with the superior overall survival in stage IV adenocarcinoma treated with a platinum-pemetrexed doublet. In advanced disease, molecular characterisation, at least in non-squamous histology, is mandatory and offers the option of targeted therapy $[14,15]$. Therefore, accurate determination of the histologic subtype of lung cancer is mandatory in stage IV. It is not yet clear for stage III $[16,17]$. In resected pulmonary carcinomas, histology can provide additional prognostic information beyond TNM staging; until now without therapeutic consequences. For pulmonary adenocarcinoma, comprehensive histologic subtyping, as established by the 2011 IASLC/American Thoracic Society/European Respiratory Society multidisciplinary classification [18] and included in the 2015 World Health Organisation classification of lung tumours [19], has been shown to have prognostic relevance independent of tumour stage. The predominant histologic subtype in resected lung adenocarcinoma correlated with overall and disease-free survival in several studies from different countries and even seems to predict benefit from adjuvant chemotherapy in a subset of patients re-evaluated in adjuvant chemotherapy trials [20]. Whether or not the predominant histologic subtype of pulmonary adenocarcinoma evaluated in biopsy specimens carries the same prognostic and predictive significance as shown for tumour resections has yet to be proven. For stereotactic body radiotherapy of the lung there is some hint that the subtype determined from core biopsies is relevant for treatment response and failure patterns [21]. As even small percentages of micropapillary and solid patterns seem to have prognostic impact $[22,23]$, the detection of any amount of these high-grade patterns in biopsy specimens should have prognostic relevance as well, but sampling error remains a major drawback especially in biopsies with small tumour volumes included [24]. Regarding pulmonary squamous cell carcinoma, no international consensus has been reached on a tumour grading system that adds prognostic information independent of tumour stage, although promising proposals exist $[25,26]$.

To date, these and other characteristics do not change the management of the patient in stage III NSCLC, but in order to get further knowledge they should be evaluated prospectively in a consequent manner.

\section{Immune features in stage III NSCLC}

In stage IV of NSCLC, immune checkpoint inhibition depending among others on programmed death-ligand (PD-L1) expression and tumour mutational burden has improved prognosis and reawakened 
interest into the immune mechanisms in lung cancer. Especially for stage III NSCLC the knowledge about immune defence and tumour stroma interactions is limited. Immunohistochemistry, partly in combination with multispectral imaging, provides some insight into the tumour stroma interaction [27]. As in other tumours one can categorise the immune activity as "inflamed" and "non-inflamed" tumours. There are data analysing tumour infiltrating lymphocytes in resected NSCLC which suggest a better survival with intense lymphocytic infiltration [28]. An analysis of published literature with the goal of evaluating subsets of tumour infiltrating lymphocytes and prognosis in patients with lung cancer suggests that high levels of CD3 + and CD8+ T-cells correlated with improved survival, but higher stromal Foxp3 levels may be detrimental [29]. There seem to be differences in resected tumours between stage I/II and III, as well between histologic tumour types especially regarding squamous and adenocarcinoma [30]. Multispectral assessment of CD8 and PD-L1 has a clear correlation with clinical outcome [31] and might have some predictive relevance in stage III NSCLC patients receiving chemoradiotherapy [32]. Neutrophil-lymphocyte ratio is a measure of systemic inflammation and was a prognostic factor for overall survival in a retrospective review of 276 patients with stage III NSCLC treated with definitive chemoradiation with or without surgery [33]. It could potentially also be an indicator of inflammatory response to radiotherapy or chemotherapy.

In the PACIFIC study, the beneficial effect of durvalumab, administered after concurrent chemoradiotherapy, was mainly observed in the PD-L1 positive subset, although these claims should be regarded as exploratory $[7,8]$. PD-L1 staining was indeed only performed in about $75 \%$ of patients and the correlation with progression-free and overall survival was based on a non-predefined subgroup of patients, of which some were very small and not corrected for imbalances. In stage IV, PD-L1 staining is only partly predictive. Other markers like tumour mutational burden and the tumour-infiltrating lymphocytes are of additional relevance. Usually there are only small biopsies in chemoradiotherapy settings which limit the analyses and interpretation. Nevertheless, the European Medicines Agency labelling for durvalumab in stage III NSCLC is based on PD-L1 expression. Therefore, it is important to obtain histology of these patients as PD-L1 staining on cytological material is feasible, but not yet widely standardised for routine implementation. Also, for PD-L1 expression on histological material, standardisation and quality assurance is very important.

In our opinion we need further prospective data in resectable and non-resectable stage III NSCLC regarding the relevance of systemic and local immune features regarding prognostic and predictive relevance.

\section{Treatment modalities}

The main local treatment modalities for patients with NSCLC in stage III are surgery and radiotherapy. As the leading site of relapse is outside the thorax, systemic treatment is usually combined with these treatments (figure 1).

\section{NSCLC stage III}

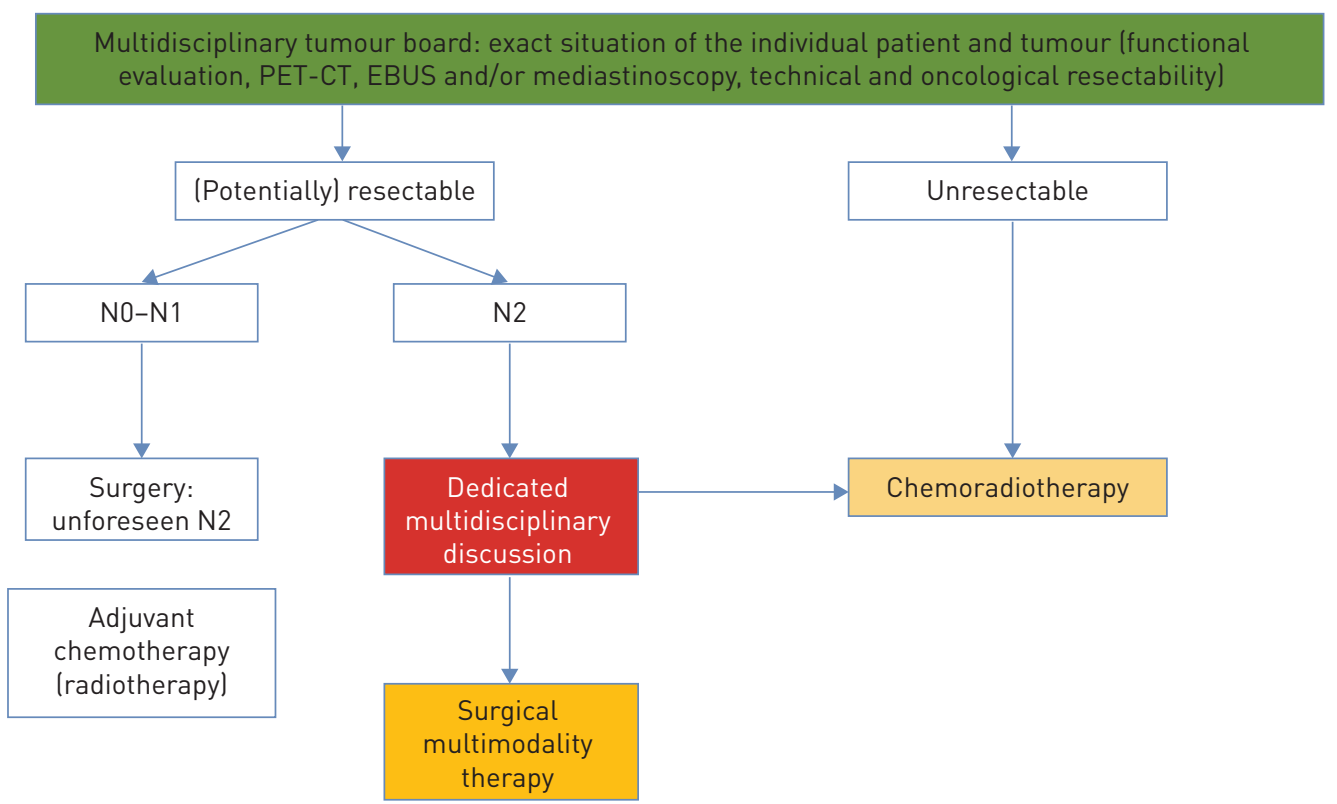

FIGURE 1 Principal management of patients with nonsmall cell lung cancer (NSCLC) in stage III. PET-CT: positron emission tomography-computed tomography; EBUS: endobronchial ultrasound. 


\section{Radiotherapy}

Concurrent treatment with platinum-based chemotherapy and thoracic radiotherapy (CCRT) is the current standard of care for patients with unresectable stage III NSCLC and recently the addition of durvalumab for 12 months after completion of CCRT. Randomised clinical trials favour the combination chemoradiotherapy compared with radiotherapy alone [34-43]. This is also the case for concomitant chemoradiotherapy compared with sequential chemoradiotherapy [44-47]. Meta-analyses of individual patient data from these studies indicate moderate but statistically significant improvements in overall survival with chemoradiotherapy versus radiotherapy alone (HR 0.89; $\mathrm{p}=0.02$ ) and with concomitant versus sequential chemoradiotherapy (HR 0.84; $\mathrm{p}=0.004$ ) $[48,49]$. In concurrent chemoradiotherapy there is an increased risk of acute, but not late, irreversible oesophageal toxicity compared with both sequential treatment and radiotherapy alone and not for pneumonitis. However, many patients are considered unsuitable for chemoradiotherapy due to poor performance status or the presence of serious comorbidities [50].

Increasing the amount of chemotherapy over two cycles as additional induction or consolidation therapy until now did not demonstrate an advantage in progression-free and overall survival in randomised trials [51-54].

Regarding systemic treatment with PD-L1 inhibition, PACIFIC is the first randomised trial which demonstrated a benefit in progression-free survival after simultaneous chemoradiotherapy with consolidation therapy using durvalumab [7, 8]. It was 16.8 months (95\% CI 13.0-18.1) with durvalumab versus 5.6 months (95\% CI 4.6-7.8) with placebo (stratified HR for disease progression or death 0.52 ; $95 \%$ CI $0.42-0.65 ; \mathrm{p}<0.001)$. Grade 3 or 4 adverse events occurred in $29.9 \%$ of the patients who received durvalumab and $26.1 \%$ of those who received placebo; the most common adverse event of grade 3 or 4 was pneumonia (4.4\% and 3.8\%, respectively). A total of $15.4 \%$ of patients in the durvalumab group and $9.8 \%$ of those in the placebo group discontinued the study drug because of adverse events. Durvalumab also significantly prolonged overall survival, as compared with placebo (stratified HR for death 0.68 ; $99.73 \%$ CI 0.47-0.997; $\mathrm{p}=0.0025$ ) [8]. Unfortunately, there are some deficits in the trial regarding, for example, the exact staging and re-staging methods and the treatment in recurrence.

The optimal strategy for radiotherapy is still under investigation. Studies have administered different radiation doses and fractions according to different schedules, including hyperfractionated accelerated radiotherapy [55-58]. In the non-concurrent setting, shortening the overall treatment time of radiotherapy led to a significant increase in 5-year overall survival [59]. In concurrent chemoradiotherapy, no regimen has been shown to be superior to $60 \mathrm{~Gy}$ delivered in 30 daily fractions of 2 Gy [60-62]. Dose escalation $>60 \mathrm{~Gy}$ in 30 daily fractions by adding 2 Gy fractions up to $74 \mathrm{~Gy}$ was detrimental for survival.

Whereas prophylactic cranial irradiation has been shown to improve survival in patients with small cell lung cancer and is a standard therapy, at least for patients with non-metastatic small cell lung cancer in complete remission [63], this is not clear for NSCLC [64-66]. Brain metastases are a common cause of relapse in patients with NSCLC, but although randomised trials consistently showed a reduction in the incidence of brain metastasis by using prophylactic cranial irradiation, this has not been translated into a gain in overall survival until now.

In conclusion, ideally patients with non-resectable stage III NSCLC should be treated in clinical trials. Outside of clinical trials for fit patients with non-resectable stage III concurrent radiochemotherapy with two cycles of cisplatinum combined with vinorelbin, pemetrexed or etoposide in our opinion is standard of care and can be followed by durvalumab in tumours which express PD-L1.

\section{Surgery}

Whereas large tumours without mediastinal lymph node involvement are usually resected, if possible, the possible role of surgery in the management of patients with stage IIIA disease with mediastinal involvement (T1-3N2M0) is the topic of considerable debate [5, 67]. Randomised controlled trials have generally found no significant difference in overall survival when surgery was compared with radiotherapy/ chemoradiotherapy for stage IIIA-N2 disease. However, a significant improvement in progression-free survival was seen with surgery versus chemoradiotherapy in one study and, in an exploratory analysis of the same study, overall survival was improved compared with chemoradiotherapy in patients who underwent lobectomy, but not pneumonectomy. This observation was not found in recent series, which also showed a lesser surgical mortality [68-70].

A number of factors are discussed in favour of a surgical approach, including single- versus multi-zone mediastinal involvement, mediastinal downstaging in response to induction chemotherapy, technical and of course functional resectability, taking into account the patient performance status including assessments of lung function, cardiac risk and exercise capacity, but all of these parameters are merely prognostic and not predictive factors. 
Two recent meta-analyses reviewed the current evidence from randomised trials addressing the role of surgery in patients with $\mathrm{N} 2$ disease as part of bimodality and trimodality treatment. [71, 72]. McELNAY et al. [71] specifically addressed the key question: should surgery be considered as part of multimodality treatment for patients with resectable lung cancer and ipsilateral mediastinal nodal disease? These systematic reviews and meta-analyses included data from six trials with a total of 868 patients. In four trials, patients received induction chemotherapy [73-76] and in two trials patients received induction chemoradiotherapy [77, 78]. The authors found no important differences in overall survival in patients randomised to surgery as part of bimodality treatment and improved overall survival in patients randomised to surgery as part of trimodality treatment in their meta-analyses. The second cumulative meta-analyses by PÖTTGEN et al. [72] reviewed randomised evidence of radiochemotherapy versus surgery within multimodality treatment in stage III NSCLC in general. Because they did not exclusively address $\mathrm{N} 2$ disease in their review they also included data from the ESPATUE trial [68] which comprised both patients with IIIA (N2) and IIIB (N3) disease. Besides that, they reviewed the same studies as McELNAY et al. [71] with one exception. They excluded the Medical Research Council trial [76] from their analysis due to relevant treatment cross-over (only four out of 24 patients were finally resected in the surgical arm). Including data from the ESPATUE trial, PöTTGEN et al. [72] found no significant different overall survival in patients with locally advanced NSCLC after induction treatment and surgery compared with those receiving definitive radiochemotherapy. Therefore, they came to a somewhat more nuanced conclusion saying that both induction therapy followed by surgery or definitive radiochemotherapy represent valuable curative treatment options for patients with stage III NSCLC, and that the individual treatment choice deserves careful interdisciplinary evaluation and counselling. In ESPATUE [68] and SAKK $[69,70]$ combining surgery with radiotherapy approximately halved the rate of local recurrences compared to surgery or radiotherapy alone.

Arguments in favour of surgery in patients with resectable disease are bulky, necrotic tumours, which are difficult to control with radiotherapy and which may lead to the formation of a lung abscess, and multiple nodules in the same lobe. The combination of preoperative concurrent chemotherapy and radiotherapy followed by surgery should be considered where local control is especially important for quality of life, such as with invasion of the brachial plexus in superior sulcus tumours (the so-called Pancoast tumours).

Finally, it should be stressed that tumours that are not resectable at diagnosis do not become resectable after any induction treatment.

In conclusion, in most patients there is no need to combine two local treatment modalities when it comes to overall survival. When local tumour control is extremely important, e.g. in case of invasion of the brachial plexus, the combination of surgery and radiotherapy is recommended and will lead to the highest chance of permanent local tumour control. Likewise, bulky, necrotic tumours especially with abscedation or with multiple cancer foci in the same lobe are better treated with surgery with or without radiotherapy. Smaller tumours with multiple nodal involvement can achieve high local cure rates with chemotherapy and radiotherapy alone. These considerations which are discussed in the multidisciplinary tumour board are outlined in table 3 .

TABLE 3 Considerations in the multidisciplinary tumour board for the various local treatment options in stage III nonsmall cell lung cancer

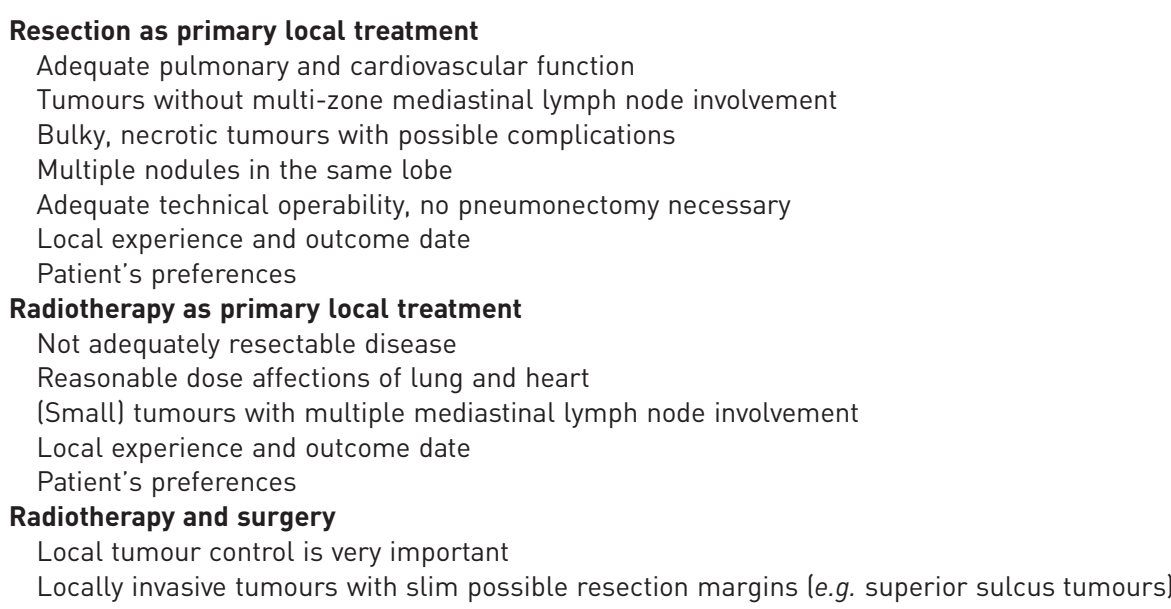


Systemic treatment

As in stage IV disease [79], both in the adjuvant/neoadjuvant as well as in the chemoradiotherapy setting, a combination of platinum-based chemotherapy with a second chemotherapeutic agent is used.

In the setting of chemoradiotherapy three major combinations are used. In North America cisplatin and etoposide or carboplatin and paclitaxel are usually applied, in Europe cisplatin and vinorelbine as well as cisplatin and etoposide are often used. There is only one randomised phase II trial which compared different combinations [80]. In this trial, a combination of induction chemotherapy with concurrent chemoradiotherapy was chosen for patients with NSCLC in stage III. The combinations which were compared were vinorelbine+cisplatin, paclitaxel+cisplatin and gemcitabine+cisplatin. In this setting efficacy was comparable, but regarding toxicity vinorelbine+cisplatin was the preferable regimen. Other direct comparisons between the combinations in the setting of chemoradiotherapy are lacking. Furthermore, on a more individualised approach including patient features, tumour characteristics like histology (squamous and non-squamous histology) and molecular patterns (driver mutations, e.g. in epidermal growth factor receptor (EGFR), EML4-ALK, etc.) are missing. If an unselected approach is chosen you may even get detrimental effects like worse survival with adjuvant gefitinib after CCRT in unselected patients [81].

In combination with surgery, either as a neoadjuvant or adjuvant approach, the most valid data are with cisplatin in combination with a taxane or, especially in the adjuvant situation, vinorelbine. There are not enough comparative data to choose the neoadjuvant or the adjuvant approach as the best option. The Spanish NATCH trial made a direct comparison of both approaches with surgery alone in a limited number of patients [82]. Under these conditions no significant difference in survival between these two approaches was found. When one looks at the other randomised phase III trials there is, in comparison to surgery alone with both approaches, a comparative survival benefit with similar toxicity [83]. In the adjuvant setting four cycles of chemotherapy are used, in the neoadjuvant setting three to four cycles are mostly used.

In conclusion, systemic therapy is an essential part of the treatment plan for patients with stage III NSCLC. Outside of clinical trials in non-resectable patients concurrent chemotherapy with two cycles of cisplatin and vinorelbine, pemetrexed (non-squamous NSCLC) or etoposide (plus adjuvant durvalumab) is used. In resectable disease neoadjuvant and adjuvant approaches are probably equivalent. But until now data for individualising systemic therapy in stage III NSCLC are largely lacking and are needed.

\section{Work in progress}

With current standard treatment, even local tumour control is suboptimal and distant metastases are common. Recurrences are often both local and at distant sites, and long-term survival remains poor. At the moment, parameters that are associated with a worse outcome are the tumour and nodal volume and the number of affected nodal stations. However, as these are prognostic factors, this knowledge will not lead to a better survival option. Therefore, molecular work-up of the tumour and immunophenotyping the tumour and its microenvironment are active fields of research. Translational and clinical trials try to integrate immunotherapeutic approaches in the perioperative setting and with chemoradiotherapy. Also targeted therapies have to be adequately included in the multimodality treatment. Stereotactic radiotherapy has to be evaluated as a component of multimodality treatment approaches especially for more advanced disease.

\section{EGFR and other driver mutations in combination with radiotherapy}

There is a good preclinical rationale for combining EGFR inhibition with radiotherapy [84-90]. Up-regulation of signalling through the EGFR pathway is implicated in various pro-oncogenic processes, including cell proliferation, invasion, survival and angiogenesis. EGFR is frequently overexpressed in patients with NSCLC $(\sim 30-80 \%$ of patients) and ionising radiation activates EGFR signalling by cancer cells potentially limiting the sensitivity to radiotherapy. Therefore, EGFR inhibitors might, independent of the mutation status, have synergistic effects when combined with radiotherapy, sensitising tumours to radiotherapy in addition to direct anti-proliferative effects. Until now the clinical data with tyrosine kinase inhibitors (TKIs) are inconclusive [91]. Unfortunately, the RTOG0617 phase III trial did not show a beneficial effect of adding cetuximab to CCRT [61, 62].

Activating somatic EGFR mutations are found in $10-20 \%$ of Caucasian patients with lung cancer. They cause constitutive activation of the EGFR tyrosine kinase. Tumours with these mutations are highly sensitive to EGFR TKI, which have been shown to be more effective than standard chemotherapy for patients with EGFR-mutated NSCLC [14]. Furthermore, NSCLC tumours with tyrosine kinase domain mutations show enhanced sensitivity to radiotherapy. Therefore, multimodality therapy including radiotherapy and a TKI might be particularly beneficial in patients with EGFR-sensitising mutations. 
However, thus far, the addition of gefitinib or erlotinib has not led to an improved survival and may even be harmful.

In the adjuvant setting, two trials in Caucasian patients were primarily performed in unselected patients where there was no benefit $[81,92]$. In one trial there was only a small percentage of patients with activating mutations and no benefit was found in this subgroup. In the other trial, the subgroup with active EGFR mutation was somewhat larger. A not statistically significant advantage for the EGFR inhibition was found, which did not result in an overall survival benefit. In a recent Chinese randomised trial in patients with activating mutation an advantage in progression-free survival could be found [17]. Overall survival data are not yet published.

Several smaller trials with EGFR tyrosine kinase inhibitors and radiotherapy in unselected patients have been reported with mixed results and usually enhanced pulmonary toxicity [93-95]. Due to the lack of specific data in stage III, chemoradiotherapy is usually also performed in patients with activating mutations reserving the TKI for the systemic relapse.

Other interventions at the molecular level in combination with thoracic radiotherapy were only done in small clinical trials. Therefore, there is uncertainty regarding safety in such combinations.

\section{Antiangiogenic agents and thoracic radiotherapy}

There are preclinical data showing that ionising radiation induces the expression of a range of proangiogenic factors, including vascular endothelial growth factor (VEGF). Radiation-induced up-regulation of signalling via the VEGF receptor (VEGFR) pathway may contribute to radiotherapy failure by enhancing the rate of vascular repair. Also the sensitisation of tumour cells to radiotherapy has been shown with both monoclonal antibodies directed against VEGFR and VEGFR-TKIs. Clinically, however, this concept was not further developed with bevacizumab as there were complications with bleeding and fistulas, especially in central tumours [96]. If the TKIs could be used, has to be evaluated. But phase I studies suggest that the risk of bleeding is also high.

\section{Immune modulation}

Even in a localised tumour the tumour stroma interaction and the status of the immune system is important. In stage IV disease the introduction of immune-checkpoint inhibitors has changed the systemic treatment. In second-line treatment, PD1/PD-L1 inhibitors are more beneficial than docetaxel [97-100]. But even in first-line, pembrolizumab is more efficacious than standard chemotherapy if $>50 \%$ of the tumours express PD-L1 [101]. Knowing the phenotypes of inflamed and non-inflamed tumours in the resected material could lead to a specific adjuvant therapy with PD1/PD-L1 inhibition. At the moment this approach is under investigation, but neoadjuvant approaches are also being tested [102]. Radiotherapy induces an inflammatory response and an upregulation of PD-L1 expression [103]. As discussed previously the addition of durvalumab to radiochemotherapy improved progression-free and overall survival. Furthermore, PD1/PD-L1 inhibitors in various combinations are studied in the context of chemoradiotherapy in clinical trials. Trials with vaccination in combination with radiotherapy or radio chemotherapy or surgery are, despite initial promising results, until now negative [104-107]. Perhaps the additional combination with PD1/PD-L1 inhibition will be efficacious. Of course the side-effect pattern has to be considered, especially the possible interaction of radiation pneumonitis and autoimmune reactions of the lung to $\mathrm{PD} 1 / \mathrm{PD}-\mathrm{L} 1$ inhibition.

\section{Specific patient groups}

\section{Elderly patients}

Overall elderly patients are not included in enough clinical trials. Therefore, our knowledge to this patient group is limited. Regarding surgery there is not a relevant limitation as long as the patients have no functional limitation, especially in lung and cardiovascular function, which exceeds the norms given in the guidelines [108].

The risk-benefit ratio of adjuvant chemotherapy in the elderly is, due to a lack of relevant data, difficult to estimate. For example, in the LACE meta-analyses the median age was 59 years in the chemotherapy arm and 60 years in the control arm with 95th percentiles of 72 and 71 years, respectively [109]. In the subgroup analysis of patients aged $\geqslant 70$ years an advantage in overall or disease-free survival could not be demonstrated. Also, radiochemotherapy data from randomised trials are sparse for elderly patients. For example, in the meta-analysis comparing concomitant versus sequential chemoradiotherapy, $13 \%$ in the concomitant arm and $19 \%$ in the sequential arm were aged $\geqslant 70$ years, the median age was 62 and 62.4 years, respectively, with a range of 79-82 years [49]. A pooled analysis of US National Cancer Institute Cooperative Group studies of concurrent chemoradiotherapy in elderly patients ( $\geqslant 70$ years) suggests a worse overall survival, more toxicity, and a higher rate of death during treatment [110]. 
Like surgery, radiotherapy alone is probably not limited by age if the functional limits are observed [108].

For chemoradiotherapy there is no clear hint on relevant negative effects of age in the subgroup analyses of the meta-analyses. But of course one has to have in mind that probably only fit elderly patients without contraindications to chemotherapy were included.

\section{Patients with poor performance status}

In the LACE meta-analysis, a small group of patients with an Eastern Cooperative Oncology Group performance status of 2 were included. In the subgroup analysis for this group no adjuvant chemotherapy was better than adjuvant chemotherapy regarding overall survival [109]. Patients with poor performance status should not receive adjuvant chemotherapy.

For example, in the meta-analysis comparing concomitant versus sequential chemoradiotherapy, $2 \%$ in the concomitant arm and $1 \%$ in the sequential arm had a performance status of 2 [49]. There is no good evidence of chemoradiotherapy being of benefit in patients with poor performance status.

\section{Interdisciplinary multimodality treatment approach}

The overall prognosis in stage III disease is rather poor with a high rate of systemic relapses. This rate varies according to the exact tumour extent and location, its histology, molecular characteristics and the immunologic situation. This setting in the individual patient can only be evaluated by a multidisciplinary team characterising the situation as a whole and planning the treatment. The outcome can be improved by adequately combining local treatments with systemic treatment concepts. Especially in N2 disease there is an overlap between surgical approaches and radiotherapy, both combined with systemic therapy. This decision is also dependent on the specific experience and the outcome in the individual centre and the preferences of the patient. Furthermore, sequencing and timing in combining these methods is relevant for the outcome of the individual patient. Overall, stage III NSCLC is the situation where interdisciplinary management is of upmost importance. There is some evidence for lung cancer in general (including stage III) that a multidisciplinary team improves various aspects of management and probably survival [111-115]. Specifically stage III is examined in a single centre study. This trial shows, for example, an improvement in mediastinal staging, unsuspected N2 disease and there is a hint for improved survival [116]. The approach to these patients varies largely and needs better evidence-based harmonisation [117]. Integrating new knowledge and new treatment strategies such as targeted therapy and immunotherapy in the management of patients in the various settings of stage III can also only be done in an interdisciplinary trial context.

\section{Conclusions}

Patients with NSCLC in stage III disease have a rather poor prognosis. Both local treatment, as well as systemic therapy, has to be optimised. Stage III disease is a heterogenous group of tumours. The outcome varies greatly and strongly depends on a good inter- and multidisciplinary strategy, involving radiologists, nuclear medicine, pathologists, molecular biology, respiratory physicians, oncology, radiotherapy and surgery. Adequately stratified interdisciplinary trials have to integrate new systemic approaches such as targeted therapy and immunotherapy.

Conflict of interest: R.M. Huber reports personal fees from AstraZeneca (Germany), Boehringer Ingelheim (Germany), BMS (Germany), Lilly, Pfizer (Germany), Roche (Germany), Takeda and MSD (Germany), outside the submitted work. D. De Ruysscher reports grants and other funding from BMS and AstraZeneca, and other funding from Roche/ Genentech, Merck/Pfizer and Celgene, during the conduct of the study. H. Hoffmann has nothing to disclose. S. Reu has nothing to disclose. A. Tufman reports personal fees from Boehringer Ingelheim, Lilly, Roche and Chugai, outside the submitted work.

\section{References}

1 Detterbeck FC, Boffa DJ, Kim AW, et al. The eighth edition lung cancer stage classification. Chest 2017; 151: 193-203.

2 Goldstraw P, Chansky K, Crowley J, et al. The IASLC Lung Cancer Staging Project: proposals for revision of the TNM stage groupings in the forthcoming (eighth) edition of the TNM classification for lung cancer. $J$ Thorac Oncol 2016; 11: 39-51.

3 Postmus PE, Kerr KM, Oudkerk M, et al. Early and locally advanced non-small-cell lung cancer (NSCLC): ESMO Clinical Practice Guidelines for diagnosis, treatment and follow-up. Ann Oncol 2017; 28: Suppl. 4, iv1-iv21.

4 Kris MG, Gaspar LE, Chaft JE, et al. Adjuvant systemic therapy and adjuvant radiation therapy for stage I to IIIa completely resected non-small-cell lung cancers: American Society of Clinical Oncology/Cancer Care Ontario Clinical Practice Guideline Update. J Clin Oncol 2017; 35: 2960-2974.

5 De Ruysscher D, Vansteenkiste J, Belderbos J, et al. The optimal local treatment of stage IIIA-N2 non-small cell lung cancer: is the issue finally settled? J Thorac Oncol 2016; 11: 284-246. 

radiation-induced abscopal effect. Trends Immunol 2018; 39: 644-655.

7 Antonia SJ, Villegas A, Daniel D, et al. Durvalumab after chemoradiotherapy in stage III non-small-cell lung cancer. N Engl J Med 2017; 377: 1919-1929.

8 Antonia SJ, Villegas A, Daniel D, et al. Overall survival with durvalumab after chemoradiotherapy in stage III NSCLC. N Engl J Med 2018; 379: 2342-2350.

9 Detterbeck FC, Boffa DJ, Tanoue LT. The new lung cancer staging system. Chest 2009; 136: 260-271.

10 Andre F, Grunenwald D, Pignon JP, et al. Survival of patients with resected N2 non-small-cell lung cancer: evidence for a subclassification and implications. J Clin Oncol 2000; 18: 2981-2989.

11 Dehing-Oberije C, De Ruysscher D, van der Weide H, et al. Tumor volume combined with number of positive lymph node stations is a more important prognostic factor than TNM stage for survival of non-small-cell lung cancer patients treated with (chemo)radiotherapy. Int J Radiat Oncol Biol Phys 2008; 70: 1039-1044.

12 Hirsch FR, Spreafico A, Novello S, et al. The prognostic and predictive role of histology in advanced non-small cell lung cancer. J Thoracic Oncol 2008; 3: 1468-1481.

13 Senan S, Brade A, Wang LH, et al. PROCLAIM: randomized phase III trial of pemetrexed-cisplatin or etoposide-cisplatin plus thoracic radiation therapy followed by consolidation chemotherapy in locally advanced nonsquamous non-small-cell lung cancer. J Clin Oncol 2016; 34: 953-962.

14 Fukuoka M, Wu YL, Thongprasert S, et al. Biomarker analyses and final overall survival results from a phase III, randomized, open-label, first-line study of gefitinib versus carboplatin/paclitaxel in clinically selected patients with advanced non-small-cell lung cancer in Asia (IPASS). J Clin Oncol 2011; 29: 2866-2874.

15 Kwak EL, Bang YJ, Camidge DR, et al. Anaplastic lymphoma kinase inhibition in non-small-cell lung cancer. N Engl J Med 2010; 363: 1693-1703.

16 Pennell NA, Neal JW, Chaft JE, et al. SELECT: a phase II trial of adjuvant erlotinib in patients with resected epidermal growth factor receptor-mutant non-small-cell lung cancer. J Clin Oncol 2019; 37: 97-104.

17 Zhong WZ, Wang Q, Mao WM, et al. Gefitinib versus vinorelbine plus cisplatin as adjuvant treatment for stage II-IIIA (N1-N2) EGFR-mutant NSCLC (ADJUVANT/CTONG1104): a randomised, open-label, phase 3 study. Lancet Oncol 2018; 19: 139-148.

18 Travis WD, Brambilla E, Noguchi M, et al. International Association for the study of Lung Cancer/American Thoracic Society/European Respiratory Society international multidisciplinary classification of lung adenocarcinoma. J Thorac Oncol 2011; 6: 244-285.

19 Travis WD, Brambilla E, Burke AP, et al. WHO Classification of Tumours of Lung, Pleura, Thymus and Heart 4th Edn. Lyon, IARC, 2015.

20 Tsao MS, Marguet S, Le Teuff G, et al. Subtype classification of lung adenocarcinoma predicts benefit from adjuvant chemotherapy in patients undergoing complete resection. J Clin Oncol 2015; 33: 3439-3446.

21 Leeman JE, Rimner A, Montecalvo J, et al. Histologic subtype in core lung biopsies of early-stage lung adenocarcinoma is a prognostic factor for treatment response and failure patterns after stereotactic body radiation therapy. Int J Radiat Oncol Biol Phys 2017; 97: 138-145.

22 Zhao Y, Wang R, Shen X, et al. Minor components of micropapillary and solid subtypes in lung adenocarcinoma are predictors of lymph node metastasis and poor prognosis. Ann Surg Oncol 2016; 23: 2099-2105.

23 Lee G, Lee HY, Jeong JY, et al. Clinical impact of minimal micropapillary pattern in invasive lung adenocarcinoma: prognostic significance and survival outcomes. Am J Surg Pathol 2015; 39: 660-666.

24 Matsuzawa R, Kirita K, Kuwata T, et al. Factors influencing the concordance of histological subtype diagnosis from biopsy and resected specimens of lung adenocarcinoma. Lung Cancer 2016; 94: 1-6.

25 Kadota K, Yumi M, Naomi K, et al. A grading system combining tumor budding and nuclear diameter predicts prognosis in resected lung squamous cell carcinoma. Am J Surg Pathol 2017; 41: 750-760.

26 Weichert W, Kossakowski C, Harms A, et al. Proposal of a prognostically relevant grading scheme for pulmonary squamous cell carcinoma. Eur Respir J 2016; 47: 938-946.

27 Mezheyeuski A, Bergsland $\mathrm{CH}$, Backman $\mathrm{M}$, et al. Multispectral imaging for quantitative and compartment-specific immune infiltrates reveals distinct immune profiles that classify lung cancer patients. J Pathol 2018; 244: 421-431.

28 Brambilla E, Le Teuff G, Marguet S, et al. Prognostic effect of tumor lymphocytic infiltration in resectable non-small-cell lung cancer. J Clin Oncol 2016; 34: 1223-1230.

29 Geng Y, Shao Y, He W, et al. Prognostic role of tumor-infiltrating lymphocytes in lung cancer: a meta-analysis. Cell Physiol Biochem 2015; 37: 1560-1571.

30 Ballesteros-Merino C, Neuberger M, Feng Z, et al. Multispectral imaging and objective assessment of immune-tumor interactions in non-small cell lung cancer. J Immunother Cancer 2015; 3: Suppl. 2, P394.

31 Stump J, Tufman A, Reu S, et al. Immunophenotyping in non-small cell lung cancer as an indicator for prognostic information and treatment decisions. Oncol Res Treat 2016; 39: Suppl. 1, 1-175.

32 Tokito T, Azuma K, Kawahara A, et al. Predictive relevance of PD-L1 expression combined with CD8+TIL density in stage III non-small cell lung cancer patients receiving concurrent chemoradiotherapy. Eur J Cancer 2016; 55: 7-14.

33 Scilla KA, Bentzen SM, Lam VK, et al. Neutrophil-lymphocyte ratio is a prognostic marker in patients with locally advanced (stage IIIA and IIIB) non-small cell lung cancer treated with combined modality therapy. Oncologist 2017; 22: 737-742.

34 Schaake-Koning C, van den BW, Dalesio O, et al. Effects of concomitant cisplatin and radiotherapy on inoperable non-small-cell lung cancer. $N$ Engl J Med 1992; 326: 524-530.

35 Blanke C, Ansari R, Mantravadi R, et al. Phase III trial of thoracic irradiation with or without cisplatin for locally advanced unresectable non-small-cell lung cancer: a Hoosier Oncology Group protocol. J Clin Oncol 1995; 13: $1425-1429$.

36 Trovo MG, Minatel E, Franchin G, et al. Radiotherapy versus radiotherapy enhanced by cisplatin in stage III non-small cell lung cancer. Int J Radiat Oncol Biol Phys 1992; 24: 11-15.

37 Jeremic B, Shibamoto Y, Acimovic L, et al. Randomized trial of hyperfractionated radiation therapy with or without concurrent chemotherapy for stage III non-small-cell lung cancer. J Clin Oncol 1995; 13: $452-458$. 
Ball D, Bishop J, Smith J, et al. A randomised phase III study of accelerated or standard fraction radiotherapy with or without concurrent carboplatin in inoperable non-small cell lung cancer: final report of an Australian multi-centre trial. Radiother Oncol 1999; 52: 129-136.

39 Jeremic B, Shibamoto Y, Acimovic L, et al. Hyperfractionated radiation therapy with or without concurrent low-dose daily carboplatin/etoposide for stage III non-small-cell lung cancer: a randomized study. J Clin Oncol 1996; 14: 1065-1070.

40 Clamon G, Herndon J, Cooper R, et al. Radiosensitization with carboplatin for patients with unresectable stage III non-small-cell lung cancer: a phase III trial of the Cancer and Leukemia Group B and the Eastern Cooperative Oncology Group. J Clin Oncol 1999; 17: 4-11.

41 Bonner JA, McGinnis WL, Stella PJ, et al. The possible advantage of hyperfractionated thoracic radiotherapy in the treatment of locally advanced nonsmall cell lung carcinoma: results of a North Central Cancer Treatment Group Phase III Study. Cancer 1998; 82: 1037-1048.

42 Groen HJ, van der Leest AH, Fokkema E, et al. Continuously infused carboplatin used as radiosensitizer in locally unresectable non-small-cell lung cancer: a multicenter phase III study. Ann Oncol 2004; 15: 427-432.

43 Belani CP, Choy H, Bonomi P, et al. Combined chemoradiotherapy regimens of paclitaxel and carboplatin for locally advanced non-small-cell lung cancer: a randomized phase II locally advanced multi-modality protocol. J Clin Oncol 2005; 23: 5883-5891.

44 Furuse K, Fukuoka M, Kawahara M, et al. Phase III study of concurrent versus sequential thoracic radiotherapy in combination with mitomycin, vindesine, and cisplatin in unresectable stage III non-small-cell lung cancer. J Clin Oncol 1999; 17: 2692-2699.

45 Fournel P, Robinet G, Thomas P, et al. Randomized phase III trial of sequential chemoradiotherapy compared with concurrent chemoradiotherapy in locally advanced non-small-cell lung cancer: Groupe Lyon-Saint-Etienne d'Oncologie Thoracique-Groupe Francais de Pneumo-Cancerologie NPC 95-01 Study. J Clin Oncol 2005; 23: 5910-5917.

46 Belderbos J, Uitterhoeve L, van Zandwijk N, et al. Randomised trial of sequential versus concurrent chemo-radiotherapy in patients with inoperable non-small cell lung cancer (EORTC 08972-22973). Eur J Cancer 2007; 43: 114-121.

47 Senan S, Cardenal F, Vansteenkiste J, et al. A randomized phase II study comparing induction or consolidation chemotherapy with cisplatin-docetaxel, plus radical concurrent chemoradiotherapy with cisplatin-docetaxel, in patients with unresectable locally advanced non-small-cell lung cancer. Ann Oncol 2011; 22: 553-558.

48 Auperin A, Le Pechoux C, Pignon JP, et al. Concomitant radio-chemotherapy based on platin compounds in patients with locally advanced non-small cell lung cancer (NSCLC): a meta-analysis of individual data from 1764 patients. Ann Oncol 2006; 17: 473-483.

49 Auperin A, Le Pechoux C, Rolland E, et al. Meta-analysis of concomitant versus sequential radiochemotherapy in locally advanced non-small-cell lung cancer. J Clin Oncol 2010; 28: 2181-2190.

50 De Ruysscher D, Botterweck A, Dirx M, et al. Eligibility for concurrent chemotherapy and radiotherapy of locally advanced lung cancer patients: a prospective, population-based study. Ann Oncol 2009; 20: 98-102.

51 Vokes EE, Herndon JE, Kelley MJ, et al. Induction chemotherapy followed by chemoradiotherapy compared with chemoradiotherapy alone for regionally advanced unresectable stage III non-small-cell lung cancer: cancer and leukemia group B. J Clin Oncol 2007; 25: 1698-1704.

52 Hanna N, Neubauer M, Yiannoutsos C, et al. Phase III study of cisplatin, etoposide, and concurrent chest radiation with or without consolidation docetaxel in patients with inoperable stage III non-small-cell lung cancer: the Hoosier Oncology Group and U.S. Oncology. J Clin Oncol 2008; 26: 5755-5760.

53 Huber RM, Flentje M, Schmidt M, et al. Simultaneous chemoradiotherapy compared with radiotherapy alone after induction chemotherapy in inoperable stage IIIA or IIIB non-small-cell lung cancer: study CTRT99/97 by the Bronchial Carcinoma Therapy Group. J Clin Oncol 2006; 24: 4397-4404.

54 Flentje M, Huber RM, Engel-Riedel W, et al. GILT - a randomised phase III study of oral vinorelbine and cisplatin with concomitant radiotherapy followed by either consolidation therapy with oral vinorelbine and cisplatin or best supportive care alone in stage III non-small cell lung cancer. Strahlenther Onkol 2016; 192: 216-222.

55 Belani CP, Wang W, Johnson DH, et al. Phase III study of the Eastern Cooperative Oncology Group (ECOG 2597): induction chemotherapy followed by either standard thoracic radiotherapy or hyperfractionated accelerated radiotherapy for patients with unresectable stage IIIA and B non-small-cell lung cancer. J Clin Oncol 2005; 23: 3760-3767.

56 Saunders M, Dische S, Barrett A, et al. Continuous, hyperfractionated, accelerated radiotherapy (CHART) versus conventional radiotherapy in non-small cell lung cancer: mature data from the randomised multicentre trial. CHART Steering committee. Radiother Oncol 1999; 52: 137-148.

57 Schild SE, Stella PJ, Geyer SM, et al. Phase III trial comparing chemotherapy plus once-daily or twice-daily radiotherapy in stage III non-small-cell lung cancer. Int J Radiat Oncol Biol Phys 2002; 54: 370-378.

58 Baumann M, Herrmann T, Koch R, et al. Final results of the randomized phase III CHARTWEL-trial (ARO 97-1) comparing hyperfractionated-accelerated versus conventionally fractionated radiotherapy in non-small cell lung cancer (NSCLC). Radiother Oncol 2011; 100: 76-85.

59 Mauguen A, Le Péchoux C, Saunders MI, et al. Hyperfractionated or accelerated radiotherapy in lung cancer: an individual patient data meta-analysis. J Clin Oncol 2012; 30: 2788-2797.

60 Bradley JD, Paulus R, Komaki R, et al. A randomized phase III comparison of standard-dose (60 Gy) versus high-dose (74 Gy) conformal chemoradiotherapy +/- cetuximab for stage IIIA/IIIB non-small cell lung cancer: preliminary findings on radiation dose in RTOG 0617. J Clin Oncol 2013; 31: Suppl. 15, 7501.

61 Bradley JD, Paulus R, Komaki R, et al. Standard-dose versus high-dose conformal radiotherapy with concurrent and consolidation carboplatin plus paclitaxel with or without cetuximab for patients with stage IIIA or IIIB non-small-cell lung cancer (RTOG 0617): a randomised, two-by-two factorial phase 3 study. Lancet Oncol 2015; 16: 187-199.

62 Bradley JD, Hu C, Komaki RU, et al. Long-term results of RTOG 0617: a randomized phase 3 comparison of standard dose versus high dose conformal chemoradiation therapy +/- cetuximab for stage III NSCLC. Int $J$ Rad Onc Biol Phys 2017; 99: S105. 

in complete remission. Prophylactic Cranial Irradiation Overview Collaborative Group. N Engl J Med 1999; 341: 476-484.

64 Gore EM, Bae K, Wong SJ, et al. Phase III comparison of prophylactic cranial irradiation versus observation in patients with locally advanced non-small-cell lung cancer: primary analysis of radiation therapy oncology group study RTOG 0214. J Clin Oncol 2011; 29: 272-278.

65 Witlox WJA, Ramaekers BLT, Zindler JD, et al. The prevention of brain metastases in non-small cell lung cancer by prophylactic cranial irradiation. Front Oncol 2018; 8: 241.

66 De Ruysscher D, Dingemans AC, Praag J, et al. Prophylactic cranial irradiation versus observation in radically treated stage III non-small-cell lung cancer: a randomized phase III NVALT-11/DLCRG-02 study. J Clin Oncol 2018; 36: 2366-2377.

67 Van Schil PE, Berzenji L, Yogeswaran SK, et al. Surgical management of stage IIIA non-small cell lung cancer. Front Oncol 2017; 7: 249.

68 Eberhardt WE, Pöttgen C, Gauler TC, et al. Phase III study of surgery versus definitive concurrent chemoradiotherapy boost in patients with resectable stage IIIA(N2) and selected IIIB non-small-cell lung cancer after induction chemotherapy and concurrent chemoradiotherapy (ESPATUE). J Clin Oncol 2015; 33: 4194-4201.

69 Pless M, Stupp R, Ris HB, et al. Induction chemoradiation in stage IIIA/N2 non-small-cell lung cancer: a phase 3 randomised trial. Lancet 2015; 386: 1049-1056.

70 Pless M, Stupp R, Ris H, et al. Final results of the SAKK 16/00 trial: a randomized phase III Trial comparing neoadjuvant chemoradiation to chemotherapy alone in stage IIIA/N2 non-small cell lung cancer (NSCLC). Ann Oncol 2014; 25: iv417.

71 McElnay PJ, Choong A, Jordan E, et al. Outcome of surgery versus radiotherapy after induction treatment in patients with N2 disease: systematic review and meta-analysis of randomised trials. Thorax 2015; 70: 764-768

72 Pöttgen C, Eberhardt W, Stamatis G, et al. Definitive radiochemotherapy versus surgery within multimodality treatment in stage III non-small cell lung cancer (NSCLC) - a cumulative meta-analysis of the randomized evidence. Oncotarget 2017; 8: 41670-41678.

73 Shepherd FA, Johnston MR, Payne D, et al. Randomized study of chemotherapy and surgery versus radiotherapy for stage IIIA non-small-cell lung cancer: a National Cancer Institute of Canada Clinical Trials Group Study. Br J Cancer 1998; 78: 683-685.

74 Johnstone DW, Byhardt RW, Ettinger D, et al. Phase III study comparing chemotherapy and radiotherapy with preoperative chemotherapy and surgical resection in patients with non-small-cell lung cancer with spread to mediastinal lymph nodes (N2); final report of RTOG 89-01. Int J Radiat Oncol Biol Phys 2002; 54: 365-369. van Meerbeeck JP, Kramer GW, Van Schil PE, et al. Randomized controlled trial of resection versus radiotherapy after induction chemotherapy in stage IIIA-N2 non-small-cell lung cancer. J Natl Cancer Inst 2007; 99: 442-450.

76 Stephens RJ, Girling DJ, Hopwood P, et al. A randomised controlled trial of pre-operative chemotherapy followed, if feasible, by resection versus radiotherapy in patients with inoperable stage T3, N1, M0 or T1-3, N2, M0 non-small cell lung cancer. Lung Cancer 2005; 49: 395-400.

77 Albain KS, Swann RS, Rusch VW, et al. Radiotherapy plus chemotherapy with or without surgical resection for stage III non-small-cell lung cancer: a phase III randomised controlled trial. Lancet 2009; 374: 379-386.

78 Sorensen JB, Riska H, Ravn J, et al. Scandinavian phase III trial of neoadjuvant chemotherapy in NSCLC stages IB-IIIA/T3. J Clin Oncol 2005; 23: Suppl. 16, 7146.

79 Schiller JH, Harrington D, Belani CP, et al. Comparison of four chemotherapy regimens for advanced non-small-cell lung cancer. N Engl J Med 2002; 346: 92-98.

80 Vokes EE, Herndon JE 2nd, Crawford J, et al. Randomized phase II study of cisplatin with gemcitabine or paclitaxel or vinorelbine as induction chemotherapy followed by concomitant chemoradiotherapy for stage IIIB non-small-cell lung cancer: cancer and leukemia group B study 9431. J Clin Oncol 2002; 20: 4191-4198.

81 Kelly K, Chansky K, Gaspar LE, et al. Phase III trial of maintenance gefitinib or placebo after concurrent chemoradiotherapy and docetaxel consolidation in inoperable stage III non-small-cell lung cancer: SWOG S0023. J Clin Oncol 2008; 26: 2450-2456.

82 Felip E, Rosell R, Maestre JA, et al. Preoperative chemotherapy plus surgery versus surgery plus adjuvant chemotherapy versus surgery alone in early-stage non-small-cell lung cancer. J Clin Oncol 2010; 28: 3138-3145.

83 Song WA, Zhou NK, Wang W, et al. Survival benefit of neoadjuvant chemotherapy in non-small cell lung cancer: an updated meta-analysis of 13 randomized control trials. J Thorac Oncol 2010; 5: 510-516.

84 Chinnaiyan P, Huang S, Vallabhaneni G, et al. Mechanisms of enhanced radiation response following epidermal growth factor receptor signaling inhibition by erlotinib (Tarceva). Cancer Res 2005; 65: 3328-3335.

85 Dittmann K, Mayer C, Fehrenbacher B, et al. Radiation-induced epidermal growth factor receptor nuclear import is linked to activation of DNA-dependent protein kinase. J Biol Chem 2005; 280: 31182-31189.

86 Pueyo G, Mesia R, Figueras A, et al. Cetuximab may inhibit tumor growth and angiogenesis induced by ionizing radiation: a preclinical rationale for maintenance treatment after radiotherapy. Oncologist 2010; 15: 976-986.

87 Das AK, Sato M, Story MD, et al. Non-small-cell lung cancers with kinase domain mutations in the epidermal growth factor receptor are sensitive to ionizing radiation. Cancer Res 2006; 66: 9601-9608.

88 Huang SM, Harari PM. Modulation of radiation response after epidermal growth factor receptor blockade in squamous cell carcinomas: inhibition of damage repair, cell cycle kinetics, and tumor angiogenesis. Clin Cancer Res 2000; 6: 2166-2174.

89 Raben D, Helfrich B, Chan DC, et al. The effects of cetuximab alone and in combination with radiation and/or chemotherapy in lung cancer. Clin Cancer Res 2005; 11: 795-805.

90 Tanaka T, Munshi A, Brooks C, et al. Gefitinib radiosensitizes non-small cell lung cancer cells by suppressing cellular DNA repair capacity. Clin Cancer Res 2008; 14: 1266-1273.

91 Ready N, Jänne PA, Bogart J, et al. Chemoradiotherapy and gefitinib in stage III non-small cell lung cancer with epidermal growth factor receptor and KRAS mutation analysis: cancer and leukemia group B (CALEB) 30106, a CALGB-stratified phase II trial. J Thorac Oncol 2010; 5: 1382-1390.

92 Goss GD, O'Callaghan C, Lorimer I, et al. Gefitinib versus placebo in completely resected non-small-cell lung cancer: results of the NCIC CTG BR19 study. J Clin Oncol 2013; 31: 3320-3326. 

radiation with or without cetuximab in patients with locally advanced unresectable non-small-cell lung cancer: Cancer and Leukemia Group B trial 30407. J Clin Oncol 2011; 29: 3120-3125.

94 Okamoto I, Takahashi T, Okamoto H, et al. Single-agent gefitinib with concurrent radiotherapy for locally advanced non-small cell lung cancer harboring mutations of the epidermal growth factor receptor. Lung Cancer 2011; 72: 199-204.

95 Wang J, Xia TY, Wang YJ, et al. Prospective study of epidermal growth factor receptor tyrosine kinase inhibitors concurrent with individualized radiotherapy for patients with locally advanced or metastatic non-small-cell lung cancer. Int J Radiat Oncol Biol Phys 2011; 81: e59-e65.

96 Spigel DR, Hainsworth JD, Yardley DA, et al. Tracheoesophageal fistula formation in patients with lung cancer treated with chemoradiation and bevacizumab. J Clin Oncol 2010; 28: 43-48.

97 Borghaei H, Paz-Ares L, Horn L, et al. Nivolumab versus docetaxel in advanced nonsquamous non-small-cell lung cancer. N Engl J Med 2015; 373: 1627-1639.

98 Brahmer J, Reckamp KL, Baas P, et al. Nivolumab versus docetaxel in advanced squamous-cell non-small-cell lung cancer. N Engl J Med 2015; 373: 123-135.

99 Herbst RS, Baas P, Kim DW, et al. Pembrolizumab versus docetaxel for previously treated, PD-L1-positive, advanced non-small-cell lung cancer (KEYNOTE-010): a randomised controlled trial. Lancet 2016; 387: $1540-1550$.

100 Rittmeyer A, Barlesi F, Waterkamp D, et al. Atezolizumab versus docetaxel in patients with previously treated non-small-cell lung cancer (OAK): a phase 3, open-label, multicentre randomised controlled trial. Lancet 2017; 389: $255-265$

101 Reck M, Rodríguez-Abreu D, Robinson AG, et al. Pembrolizumab versus chemotherapy for PD-L1-positive non-small-cell lung cancer. N Engl J Med 2016; 375: 1823-1833.

102 Forde PM, Chaft JE, Smith KN, et al. Neoadjuvant PD-1 blockade in resectable lung cancer. N Engl J Med 2018; 378: 1976-1986.

103 Schoenhals JE, Seyedin SN, Anderson C, et al. Uncovering the immune tumor microenvironment in non-small cell lung cancer to understand response rates to checkpoint blockade and radiation. Transl Lung Cancer Res 2017; 6: 148-158.

104 Butts C, Maksymiuk A, Goss G, et al. Updated survival analysis in patients with stage IIIB or IV non-small-cell lung cancer receiving BLP25 liposome vaccine (L-BLP25): phase IIB randomized, multicenter, open-label trial. J Cancer Res Clin Oncol 2011; 137: 1337-1342.

105 Vansteenkiste JF, Cho BC, Vanakesa T, et al. Efficacy of the MAGE-A3 cancer immunotherapeutic as adjuvant therapy in patients with resected MAGE-A3-positive non-small-cell lung cancer (MAGRIT): a randomised, double-blind, placebo-controlled, phase 3 trial. Lancet Oncol 2016; 17: 822-835.

106 Mitchell P, Thatcher N, Socinski MA, et al. Tecemotide in unresectable stage III non-small-cell lung cancer in the phase III START study: updated overall survival and biomarker analyses. Ann Oncol 2015; 26: 1134-1142.

107 National Cancer Institute (NCI). Vaccine therapy, chemotherapy, and radiation therapy in treating patients with stage III non-small cell lung cancer that cannot be removed with surgery. http://clinicaltrials.gov/ct2/show/ NCT00091039 Date last updated: February 6, 2009. Date last accessed: July 6, 2012.

108 Brunelli A, Charloux A, Bolliger CT, et al. ERS/ESTS clinical guidelines on fitness for radical therapy in lung cancer patients (surgery and chemo-radiotherapy). Eur Respir J 2009; 34: 17-41.

109 Pignon J-P, Tribodet H, Scagliotti GV, et al. Lung adjuvant cisplatin evaluation: a pooled analysis by the LACE collaborative group. J Clin Oncol 2008; 26: 3552-3559.

110 Stinchcombe TE, Zhang Y, Vokes EE, et al. Pooled analysis of individual patient data on concurrent chemoradiotherapy for stage III non-small-cell lung cancer in elderly patients compared with younger patients who participated in US National Cancer Institute Cooperative Group Studies. J Clin Oncol 2017; 35: 2885-2892.

111 Boxer MM, Vinod SK, Shafiq J, et al. Do multidisciplinary team meetings make a difference in the management of lung cancer? Cancer 2011; 117: 5112-5120.

112 Schmidt HM, Roberts JM, Bodnar AM, et al. Thoracic multidisciplinary tumor board routinely impacts therapeutic plans in patients with lung and esophageal cancer: a prospective cohort study. Ann Thorac Surg 2015; 99: $1719-1724$

113 Takeda T, Takeda S, Uryu K, et al. Multidisciplinary lung cancer tumor board connecting eight general hospitals in Japan via a high-security communication line. JCO Clin Cancer Inform 2019; 3: 1-7.

114 Bilfinger TV, Albano D, Perwaiz M, et al. Survival outcomes among lung cancer patients treated using a multidisciplinary team approach. Clin Lung Cancer 2018; 19: 346-351.

115 Freeman RK, Van Woerkom JM, Vyverberg A, et al. The effect of a multidisciplinary thoracic malignancy conference on the treatment of patients with lung cancer. Eur J Cardiothorac Surg 2010; 38: 1-5.

116 Friedman EL, Kruklitis RJ, Patson BJ, et al. Effectiveness of a thoracic multidisciplinary clinic in the treatment of stage III non-small-cell lung cancer. J Multidiscip Healthc 2016; 9: 267-274.

117 Dickhoff C, Dahele M, Smit EF, et al. Patterns of care and outcomes for stage IIIB non-small cell lung cancer in the TNM-7 era: results from the Netherlands Cancer Registry. Lung Cancer 2017; 110: 14-18. 